\title{
Ultraviolet Characteristics of a Silicon Inversion Layer for Applications in Radiometry
}

\author{
Pearson V.C. Luhanga \\ Physics Department, University of Botswana, Gaborone, Botswana
}

\begin{abstract}
The inversion-layer (IL) that can be induced in a silicon surface shows very good sensitivity and spectral response in the ultraviolet $(U V)$ region of electromagnetic radiation. As such, it is possible to use this structure as a relatively cheap radiometer for $U V$ radiation measurements. The fabrication process for such a device is shown to be relatively cheaper than that of a diffused pn-junction, which is the typical design for a photocell. Hence the construction of a relatively cheap UV radiometer is possible.
\end{abstract}

Keywords: ultraviolet, radiometry, silicon inversion layer, photocell

\section{Introduction}

The ultraviolet radiation is the high quantum energy radiation ranging in wavelength from approximately 400 nanometers $(\mathrm{nm})$ at end of the visible spectrum to approximately $40 \mathrm{~nm}$ at the X-ray end. The solar UV radiation to be discussed here is in the wavelength range of $200 \mathrm{~nm}$ to $400 \mathrm{~nm}$. The associated energies of this type of radiation is such that it gets absorbed by most forms of matter including living tissue. In living tissue, such high energy photons may excite electrons, or even eject them from the atoms or molecules, hence causing ionization. Due to this ionization potential, exposure to ultraviolet radiation has been found to cause skin cancers (e.g. basal-cell and squamous-cell carcinoma and melanoma), cataracts of the eyes, wrinkling of the skin, suppression of the immune system, and sunburn, in people. The high energy radiation deranges the molecular structures of the nucleic acids DNA and RNA and proteins, causing the above problems.

The sun is a strong source of ultraviolet radiation, but very little of it reaches the earth; most of it being absorbed by atmospheric gases, chiefly ozone. Therefore a depletion of the ozone layer of the atmosphere could cause very serious problems to life on earth.

Ozone measurements were started at British Antarctic base at Halley Bay in 1956. The ozone level was found to fall quite appreciableduring the months of September to October, to about 150 Dobson Units (DU) which is about half the normal level there. In 1983 the scientists working with this British Antarctic survey discovered a hole in the ozone layer over the Antarctica, which showed that the ozone over the Halley Bay has been falling since the 1960's Gribbin[1]. Chehade et.al. [2], and Arblaster, J.M., [3] have also shown this trend in the decrease in the ozone over the period 1979-2012.

It was discovered that the ozone depleting substances were mostly man-made, chiefly chloro-fluorocarbons (CFCs); which are used as coolants in refrigerators and air conditioners, and also as propellants in aerosol cans. A majority of the refrigerant coolants contain various combinations of chemicals such as chlorine, fluorine, bromine, carbon and hydrogen. The chief cause of the depletion of the ozone layer is due to the release of chlorine from these CFCs.

The concern about the earth's ozone depletion lead to the signing of the Montreal protocol in 1987. This was to restrict the production, sale and consumption of CFCs. By 1995 most developed countries had stopped the production of CFCs.

The decrease in the ozone layer in the atmosphere, by the compounds discussed above, can lead to an increase in the solar ultraviolet radiation reaching the earth, thereby leading to the very serious consequences on all life on earth as discussed above. Therefore it is very important that the ultraviolet radiation reaching the earth is monitored. Hence there is need to develop low-cost radiometers with high sensitivity in the ultraviolet spectrum.

\section{Measurement of Solar Ultraviolet Radiation}

Measurement of solar ultraviolet radiation at ground level is very important, but it is limited by the steep reduction of this radiation below the wavelength of $320 \mathrm{~nm}$ at ground level due to the absorption by the ozone layer of the atmosphere. Pyranometers which are fitted with appropriate wavelength filters can be used to measure this radiation at ground level, but these instruments are very expensive; typically at $\$ 2000$, without such filters. The other problem with such instruments is their low sensitivity, typically $10 \mu \mathrm{V} / \mathrm{W} \cdot \mathrm{m}^{-2}$ for thermopile types (for the visible range of solar radiation). This makes such instruments unsuitable for the measurement of solar ultraviolet radiation because this radiation is very low, and the wavelength filters would reduce the sensitivity even further.

Nowadays most of the instruments used for measurement of ultraviolet radiation are based on the use of a fluorescent phosphor, which changes the ultraviolet radiation below about $320 \mathrm{~nm}$ into visible light of 400-600 $\mathrm{nm}$ wavelengths. This visible light is then measured with a photomultiplier, Frohlich [4].Eppley Laboratories of Newport, R.I., USA do make an ultraviolet radiometer (TUVR) for the wavelength range of 295-385 $\mathrm{nm}$. This device uses a selenium barrierlayer photocell to increase the sensitivity to about 150 $\mu \mathrm{V} / \mathrm{W} \cdot \mathrm{m}^{-2}$, and also uses a quartz widow diffuser to enhance the cosine response [5]. But here again we find that this type 


\section{International Journal of Science and Research (IJSR) \\ ISSN (Online): 2319-7064}

Index Copernicus Value (2013): 6.14 | Impact Factor (2015): 6.391

of instrument is very expensive, typically US\$2000.Kipp\&Zonen also manufacture ultraviolet radiometers, their model CUV5 total ultraviolet radiometer costs more than US\$3000, Campbell Scientific[6], and Omni Instruments [7].

To cut down the cost of solar ultraviolet radiometry, we need to seek a low-cost sensor. This can then be implemented in instruments that are used to measure ultraviolet radiation. An implicitly cheap sensor but very sensitive to ultraviolet radiation is the silicon-inversion layer diode. The theoretical spectral response of this device in the UV region is fairly uniform. Its sensitivity starts to drop as the wavelength is increased beyond the visible range, and falls off quite sharply as the wavelength increases into the infrared. When the radiation wavelength is greater than 1100 $\mathrm{nm}$ the device is almost transparent to the radiation. This is not a problem as the device is to be used as a sensor only for UV radiation.

\section{Spectral Characteristics of pn-Junctions}

\section{The Diffused pn-junction}

The sensor that is chiefly used in low-cost instruments for visible radiation is the diffused pn junction diode. The diffused pn junction's sensitivity dropsas the wavelength gets reduced into the blue region $[8,9,10]$.

Series BPX65 - Typical Spectral Response

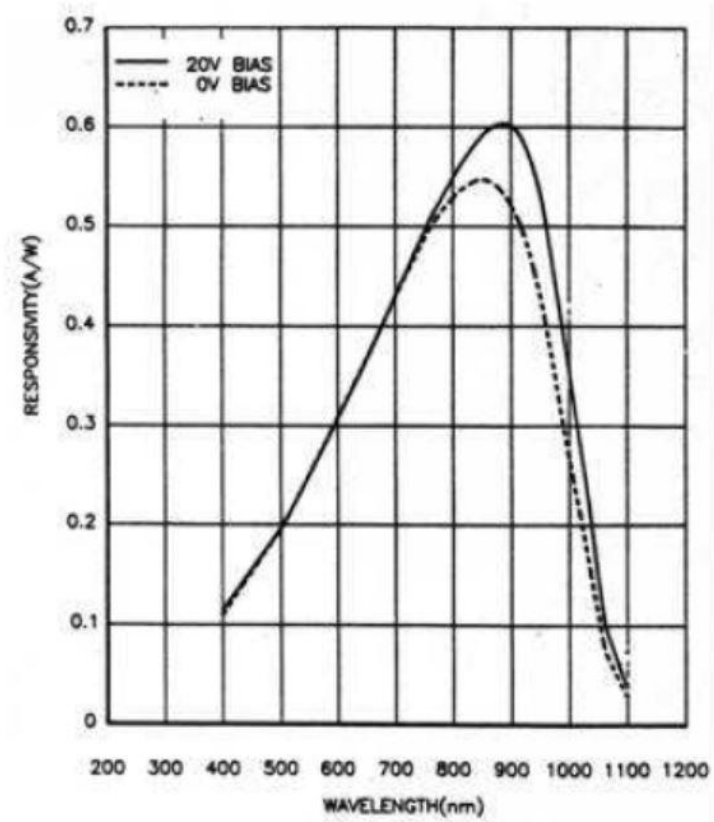

Figure 1: Spectral response of BPX65 diffused pn-junction photodiode $[11,12]$

Therefore, the pn junction is not suitable for UV radiometry. Techniques have been employed with the diffused pn junction, to improve on its sensitivity in the ultraviolet range of radiation so that the device can be employed in UV radiometry, e.g..Vanyushin et al. [13], Wataru Kubo, et al. [14].

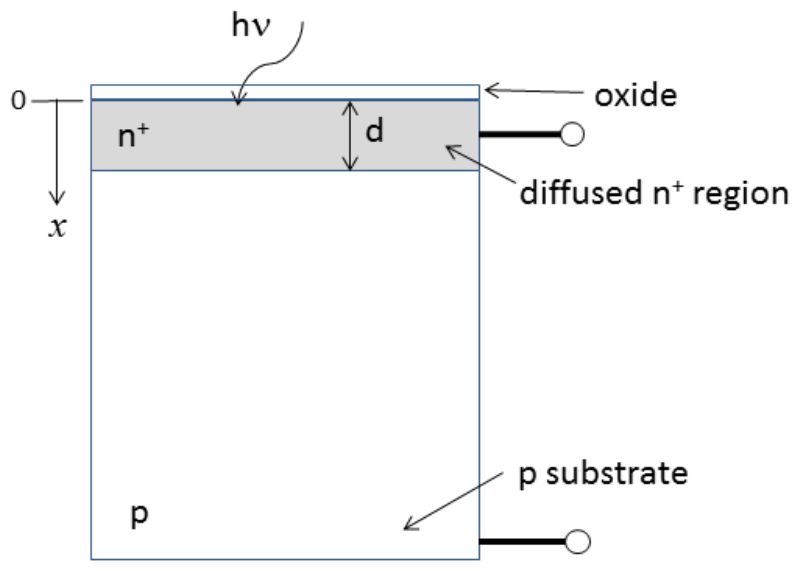

Figure 2: Structure of a diffused pn-junction(DJ) photodiode

The structure of a diffused pn-junction photocell is shown in figure 2 , the thickness of the diffused $\mathrm{n}^{+}$layer being $d$. The structure is modelled as a vertical collection onedimensional device. The device is illuminated from $x=0$ origin. The continuity equation is thus given by

$$
D_{n} \frac{d^{2} n}{d x^{2}}+G_{L}-\frac{n_{p}-n_{p_{0}}}{\tau_{n}}=0
$$

for electrons in the p-region, and a similar expression for holes in the n-region. The generation rate $G_{L}$ is given by

$$
G_{L}=\alpha(\lambda) \phi \exp (-\alpha(\lambda) x)
$$

where $\alpha$ is the absorption coefficient of the semiconductor and is a function of the wavelength $\lambda$ of the illumination, Dash et.al [15], Rajkanan et. al [16]; and $\phi$ is the incident spectral radiant-flux density.

If we assume low-level injection of minority carriers and a semi-infinite substrate thickness, we can use the above equations to find the number of carries that cross the pn junction and contribute to the external photocurrent, $J_{p h}$ as

$$
J_{p h}=q \phi\left[\frac{\alpha L_{p}}{1-\alpha L_{p}}\left(e^{\alpha d}-e^{-d / L_{p}}\right)+\frac{\alpha L_{n}}{1+\alpha L_{n}} e^{-\alpha d}\right]
$$

where $L_{p}$ is the hole diffusion length in the diffused $\mathrm{n}^{+}$ region, $L_{n}$ is the electron diffusion length in the p-substrate, and $d$ is the junction depth(see Fig. 2).

\section{The Inversion-Layer (IL) pn-junction}

The principle involved in the Inversion-layer photodiode is to use the inversion layer that is induced at the silicon surface by the charges in the oxide insulator of the oxidesemiconductor structure, as one side of a field-induced pn junction diode. 


\section{International Journal of Science and Research (IJSR) \\ ISSN (Online): 2319-7064}

Index Copernicus Value (2013): 6.14 | Impact Factor (2015): 6.391

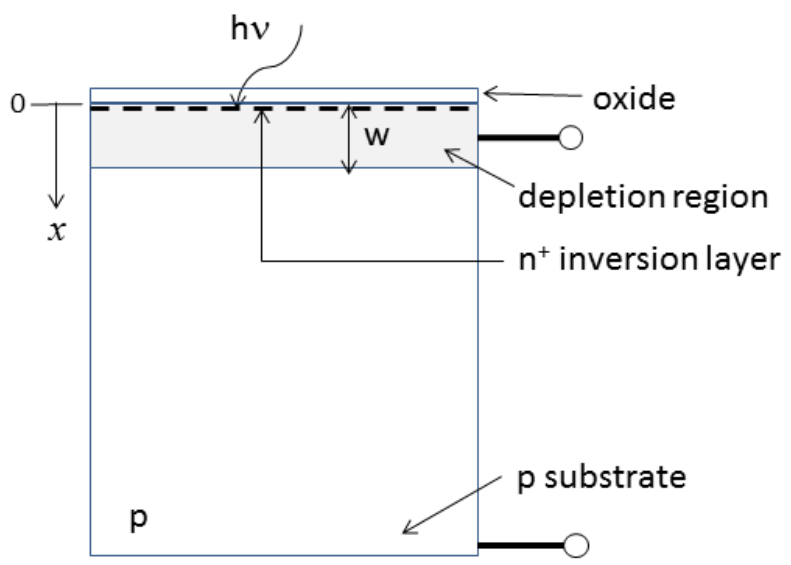

Figure 3: Structure of an Inversion-layer (IL) photodiode

The fabrication process of this structure is very simple and cheap. It involves a low-temperature growth of thermal oxide at the silicon surface. Thus when this structure is used as the sensor in a photocell, it will make the fabrication process of the photocell very cheap indeed.

Using equations (1) and (2) above we can once again derive the photocurrent density of this device to be (Gartner [18], Brown et. al [19], Salter et al. [20]):

$$
J_{p h[I L]}=q \phi\left[1-\frac{e^{-\alpha W}}{1+\alpha L_{n}}\right]
$$

where $W$ is the width of the depletion layer at the surface of the semiconductor. Calculated spectral characteristics of the two structures (the diffused pn junction and the inversionlayer pn junction) using equations (3) and (4) are shown in figure 4 . This graph is a plot of the normalized photocurrent density $\left(\mathrm{J}_{\mathrm{ph}} / \mathrm{q} \phi\right)$ as a function of wavelength. It has been plotted using the absorption coefficient data from Rajkanan et al [16] and Terman[17]; and by making the diffusion junction depth $d$ equal to the inversion layer width $w$ (i.e. $d=$ $w$ ), and making $L_{n}$ the same for both devices.

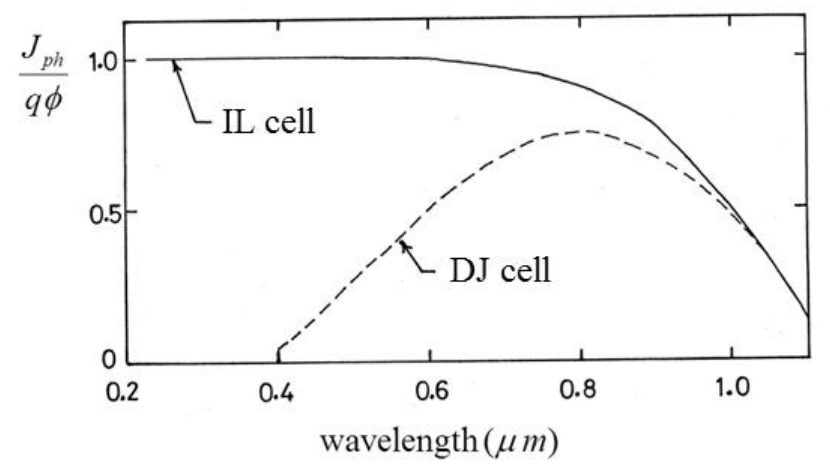

Figure 4: Comparison of the calculated spectral characteristics of the DJ and the IL photocells.

Figure 4 shows clearly the better sensitivity of the inversionlayer photocell compared with the diffused junction photocell. The higher ultraviolet photosensitivity of the inversion layer photocell is mainly due to low recombination losses at the surface, since normally recombination rates are lower at a thermally oxidized silicon surface than at a diffused surface, Salter et. al [20]. Secondly, since diffusion induced damage is not present in the inversion layer structure, the minority carrier lifetimes should be higher than in the diffused junction structures, thus leading to higher photocurrent. Salter et al. also found that the inversion layer pn junction diode is less susceptible to lifetime and surface recombination velocity variations than the diffued junction diode, thus making the inversion layer structure more effective in collecting carriers generated by ultraviolet radiation. These superior characteristics of the inversion layer photocell in the ultraviolet region compared with the diffused junction photocell have been demonstrated experimentally by Gorban et al. [21] and Gedfrey et al. [22].

\section{Conclusions}

In the analysis above we have shown that the inversion layer photocell shows intrinsically that it has a very high sensitivity in the ultraviolet radiation range, which is fairly uniform over the entire ultraviolet range. It has also been shown that the fabrication process of such a photocell is very simple and cheap. We therefore conclude that the inversion layer structure can be used as a low-cost, but very high sensitivity sensor for ultraviolet radiation measurement. It is much cheaper than the sensors that are used currently in UV radiometers.

\section{References}

[1] Gribbin, J., The ozone layer, New Scientist: Inside Science vol. 9, 1-4, May 5, 1988.

[2] W. Chehade, M.Weber, and J.P. Burrows; Total ozone trends and variability during 1979-2012 from merged data sets of various satellites. Atmos. Chem. Phys., 14, 7059-7074, 2014.

[3] Arblaster, J.M., and N.P Gillett (Lead Authors), N. Calvo, P.M. Forster, L.M. Polvani, S.-W. Son, D.W.Waugh, and P.J. Young, Stratospheric ozone changes and climate, Chapter 4 in Scientific Assessment ofOzone Depletion: 2014, Global Ozone Research and Monitoring Project - Report No. 55, WorldMeteorological Organization, Geneva, Switzerland, 2014.

[4] Frohlich, C., and London, J. (Editors); Revised Instruction Manual on Radiation Instruments and Measurements, World Climate Research Programme (WCRP),October 1986.

[5] http://www.eppleylab.com/instrumentation/total_ultravi olet_radiometer.ht; last accessed on June $20^{\text {th }}, 2016$.

[6] Campbell Scientific Price List, 2014

[7] Omni Instruments Price List, http://www.omniinstruments.co.uk/weather-stationsand-instruments/radiometers-uv-ir-net-solarirradiance.html, http://www.omniinstruments.co.uk/cuv5-ultra-violetradiometer.html; accessed June 20, 2016.

[8] Bentham Instruments Ltd, UK; Spectral characteristics of photovoltaic devices, Technical Note, 2016.

[9] Arnaud Darmont, Aphesa (www.aphesa.com), White paper, April 2009; Spectral Response of Silicon Image Sensors. 2009

[10] PV Education - $\quad$ Spectral Response; http://www.pveducation.org/pvcdrom/solar-celloperation/spectral-response

\section{Volume 5 Issue 7, July 2016




\section{International Journal of Science and Research (IJSR) \\ ISSN (Online): 2319-7064}

Index Copernicus Value (2013): 6.14 | Impact Factor (2015): 6.391

[11]Farnell/Newark Data sheet; www.centronic.co.uk

[12] RS Components Data sheet 298-4562

[13] Vanyushin, I.V. et al., Adjusting the spectral response of silicon phtotdiodes by additional dopant implantation. Russian Microelectronics, vol. 34, No. 3, 155-159, 2005.

[14] Wataru Kubo, et. al., Dye-sensitized solar cells: improvement of spectral response by tandem structure, Journal of Photochemistry and Photobiology A: Chemistry, vol 164, 33-39, 2004.

[15]Dash, W.C. and Newman, R., Intrinsic optical absorption in single-crystal germanium and silicon at 77 $\mathrm{K}$ and $300 \mathrm{~K}$, Physical Review, 99(4), 1151-1155, 1955.

[16] Rajkanan, K., Singh, R. and Shewchun, J., Absorption coefficients of silicon for solar cell calculations, Solidstate Electronics, 22, 792-793, 1978.

[17] Terman, L.M., Spectral response of solar-cell structures, Solid-state Electronics, 2(1), 1-7, 1961.

[18] Gartner, W.W., Depletion-layer photoeffects in semiconductors, Physical Review, 116(1), 84-87, 1959.

[19]Brown, R.W. and Chamberlain, S.G., Quantum efficiency of a silicon gate charge-coupled optical imaging array, Phy. stat. sol. (a), 20, 675-685, 1973.

[20] Salter, G.C. and Thomas, R.E., Silicon solar cells using natural inversion layers found in thermally oxidized psilicon. Solid-state Electronics, 20, 95-104, 1977.

[21] Gorban, A.P., Litovchenko, V.G., Popov, V.G., and Serba, A.A., Photo-electric characteristics of surfacechannel photocells. Sov. Phys. Semicond., 11(7), 824825, 1977.

[22] Godfrey, R.B., Green, M.A., $655 \mathrm{mV}$ open-circuit voltage, $17.6 \%$ efficient silicon MIS solar cells. Appl. Phys. Lett., 34(11), 790-793, 1979. 